\title{
A SPACE WHICH CONTAINS NO REALCOMPACT DENSE SUBSPACE
}

\author{
TOSHIJI TERADA
}

\begin{abstract}
A Tychonoff space which contains no realcompact space as a dense subspace is constructed. Let $\mathscr{D} Q R$ be the class of all spaces which contain some realcompact spaces as dense subspaces. Then, as a consequence of the above result, it follows that $\mathscr{D} R$ is not closed-hereditary.
\end{abstract}

All spaces considered here are Tychonoff topological spaces. Let $\mathscr{Q} R$ be the class of all spaces which contain some realcompact spaces as dense subspaces. In [5], the author noticed that the class $\mathscr{Q} \mathscr{R}$ is closed-hereditary if and only if $\mathscr{Q} \Re$ is the class of all spaces. If we assume the existence of a measurable cardinal, then each discrete space of a measurable cardinal cannot contain a realcompact space as a dense subspace, and hence $\mathscr{D} R$ is not closed-hereditary. However, without any additional set theoretical assumption, it is not clear whether $\mathscr{Q} \mathcal{R}$ is closed-hereditary.

In this note we shall give naively a space which contains no realcompact space as a dense subspace. Hence it follows that $\mathscr{D} R$ is not closed-hereditary.

A space is called $\mathrm{Oz}$ (or perfectly $\kappa$-normal) if every regular closed subset is a zero-set (see $[2,3,4])$. If $X$ is $\mathrm{Oz}$, then every dense subspace is $z$-embedded in $X$. Ščepin showed that every product of metrizable spaces is Oz. Blair and Hager [1] showed that a $z$-embedded subspace $Y$ of a realcompact space $X$ is realcompact if and only if $Y$ is $G_{\delta}$-closed in $X$ (i.e. for any point $x$ in $X-Y$ there is a $G_{\delta}$-set $S$ of $X$ such that $x \in S$ and $S \cap Y=\varnothing$ ). From this fact, the following lemma is obvious.

Lemma 1. Let $Y$ be a dense subspace of a realcompact Oz-space $X$. Then the following are equivalent.

(1) $Y$ is realcompact.

(2) $Y$ is $G_{\delta}$-closed in $X$.

Let $A$ be a set such that $|A| \geqslant \boldsymbol{\aleph}_{1}$. For each $\alpha$ in $A$ let $X_{\alpha}$ be the space of rational numbers. Let $p_{\alpha}=0, q_{\alpha}=1$ in $X_{\alpha}$ and let $X_{\alpha}^{\prime}=X_{\alpha}-\left\{p_{\alpha}, q_{\alpha}\right\}$. The family of all nonempty finite subsets of $A$ is denoted by $\mathscr{F}(A)$. Let $\mathcal{E}$ be a disjoint family of countably infinite subsets of $A$ such that $|\mathcal{E}|=|A|$. Since the cardinality of the set

Received by the editors April 26, 1982 and, in revised form, August 3, 1982.

1980 Mathematics Subject Classification. Primary 54D60, 54G20; Secondary 54B05, 54B10.

Key words and phrases. Dense subspace, realcompact, $G_{\delta}$-closed, $\mathrm{Oz}, \sigma$-product.

(c) 1983 American Mathematical Society 0002-9939/82/0000-0943/\$01.50 
$\cup\left\{\Pi\left\{X_{\beta}^{\prime}: \beta \in B\right\}: B \in \mathscr{F}(A)\right\}$ is equal to that of $A$, there is a one-to-one map $t$ : $\cup\left\{\Pi\left\{X_{\beta}^{\prime}: \beta \in B\right\}: B \in \mathscr{F}(A)\right\} \rightarrow \mathcal{E}$. For each $B$ in $\mathscr{F}(A)$ we define a map $h_{B}$ : $\Pi\left\{X_{\beta}^{\prime}: \beta \in B\right\} \rightarrow \Pi\left\{X_{\alpha}: \alpha \in A\right\}$ in the following way;

$$
\pi_{\alpha}\left(h_{B}\left(\left\langle y_{\beta}\right\rangle\right)\right)= \begin{cases}y_{\alpha} & \text { if } \alpha \in B, \\ q_{\alpha} & \text { if } \alpha \in t\left(\left\langle y_{\beta}\right\rangle\right)-B, \\ p_{\alpha} & \text { otherwise, }\end{cases}
$$

where $\pi_{\alpha}: \Pi\left\{X_{\alpha}: \alpha \in A\right\} \rightarrow X_{\alpha}$ is the natural projection and $\left\langle y_{\beta}\right\rangle$ means a member of $\Pi\left\{X_{\beta}^{\prime}: \beta \in B\right\}$ whose $\beta$-coordinate is $y_{\beta}$. Let $Z_{A}$ be the subspace of $\Pi\left\{X_{\alpha}: \alpha \in A\right\}$ defined by $\cup\left\{h_{B}\left(\Pi\left\{X_{\beta}^{\prime}: \quad \beta \in B\right\}\right): B \in \mathscr{F}(A)\right\}$. Obviously $Z_{A}$ is dense in $\Pi\left\{X_{\alpha}: \alpha \in A\right\}$. In the rest of this note, for the sake of simpiicity, $\Pi\left\{X_{\alpha}: \alpha \in A\right\}$ is denoted by $X_{A}$. If $\left\langle x_{\alpha}\right\rangle=h_{B}\left(\left\langle y_{\beta}\right\rangle\right)$, then $\left\langle y_{\beta}\right\rangle$ is denoted by $s\left(\left\langle x_{\alpha}\right\rangle\right)$ and $B$ is denoted by $i\left(\left\langle x_{\alpha}\right\rangle\right)$. Countable intersections of canonical open subsets of $X_{A}$ are called canonical $G_{\delta}$-sets.

Lemma 2. The density of $Z_{A}$ is not less than $\aleph_{1}$.

Proof. Assume that there is a dense subspace $Y$ in $Z_{A}$ such that $|Y| \leqslant \aleph_{0}$. Note that $Y$ is also dense in $X_{A}$. For each point $\left\langle x_{\alpha}\right\rangle$ in $Y$ the set $\left\{\alpha \in A: x_{\alpha} \neq p_{\alpha}\right\}$ is countable. Let $A_{\left\langle x_{\alpha}\right\rangle}=\left\{\alpha \in A: x_{\alpha} \neq p_{\alpha}\right\}$. Then the cardinality of the set $A^{\prime}=$ $\cup\left\{A_{\left\langle x_{\alpha}\right\rangle}:\left\langle x_{\alpha}\right\rangle \in Y\right\}$ is not more than $\boldsymbol{\aleph}_{0}$. Hence there is a member $\alpha_{0}$ in $A-A^{\prime}$. This implies that the $\alpha_{0}$-coordinate of every member of $Y$ is $p_{\alpha_{0}}$. But this shows that $Y$ is not dense in $X_{A}$. This is a contradiction.

ProOF. $Z_{A}$ contains no realcompact space as a dense subspace.

Proof. Assume that $Z_{A}$ contains a realcompact space $Y$ as a dense subspace. Since $Y$ is dense in the realcompact Oz-space $X_{A}, Y$ must be $G_{\delta}$-closed in $X_{A}$ by Lemma 1. Let $\sigma_{A}$ be the $\sigma$-product of $\left\{X_{\alpha}: \alpha \in A\right\}$ with the base point $\left\langle p_{\alpha}\right\rangle$. Notice that $\sigma_{A} \cap Z_{A}=\varnothing$. Let $\left\langle x_{\alpha}\right\rangle$ be an arbitrary point of $\sigma_{A}$. Then there is a canonical $G_{\delta}$-set $S_{\left\langle x_{\alpha}\right\rangle}$ in $X_{A}$ such that $\left\langle x_{\alpha}\right\rangle \in S_{\left\langle x_{\alpha}\right\rangle}$ and $S_{\left\langle x_{\alpha}\right\rangle} \cap Y=\varnothing$. We can assume that $S_{\left\langle x_{\alpha}\right\rangle}=\Pi\left\{S_{x_{\alpha}}: \alpha \in A\right\}$ where $S_{x_{\alpha}}$ is a $G_{\delta}$-set in $X_{\alpha}$ and $S_{x_{\alpha}}=X_{\alpha}$ except countably many $\alpha$ 's. Let $B_{\left\langle x_{\alpha}\right\rangle}=\left\{\alpha \in A: x_{\alpha} \neq p_{\alpha}\right\}$ and let $C_{\left\langle x_{\alpha}\right\rangle}=\left\{\alpha \in A: S_{x_{\alpha}} \neq X_{\alpha}\right\}$. Note that $B_{\left\langle x_{\alpha}\right\rangle}$ is finite and $C_{\left\langle x_{\alpha}\right\rangle}$ is countable. Now, we construct a sequence $\left\{C_{n}\right.$ : $n=0,1,2, \ldots\}$ of subsets of $A$ in the following way. Let $C_{0}$ be an arbitrary nonempty countable subset of $A$. Assume that $C_{n}$ is constructed such that $\left|C_{n}\right| \leqslant \boldsymbol{\aleph}_{0}$. Let $\sigma_{A}^{n}=\left\{\left\langle x_{\alpha}\right\rangle \in \sigma_{A}: B_{\left\langle x_{\alpha}\right\rangle} \subset C_{n}\right\}$. Then the cardinality of $\sigma_{A}^{n}$ is at most $\boldsymbol{\aleph}_{0}$. Let $C_{n+1}=C_{n} \cup \cup\left\{C_{\left\langle x_{\alpha}\right\rangle}:\left\langle x_{\alpha}\right\rangle \in \sigma_{A}^{n}\right\}$. It is obvious that $\left|C_{n+1}\right| \leqslant \boldsymbol{\aleph}_{0}$. Let $C=$ $\cup\left\{C_{n}: n=0,1,2, \ldots\right\}$. Then obviously $|C| \leqslant \boldsymbol{\kappa}_{0}$. We shall show that $t\left(s\left(\left\langle x_{\alpha}\right\rangle\right)\right) \cap$ $C \neq \varnothing$ for each element $\left\langle x_{\alpha}\right\rangle$ of $Y$. Assume that $t\left(s\left(\left\langle x_{\alpha}\right\rangle\right)\right) \cap C=\varnothing$. Since $i\left(\left\langle x_{\alpha}\right\rangle\right)$ is finite, $i\left(\left\langle x_{\alpha}\right\rangle\right) \cap C \subset C_{n}$ for some $n$. Let $\left\langle z_{\alpha}\right\rangle$ be the element of $\sigma_{A}$ determined in the following way:

$$
z_{\alpha}= \begin{cases}x_{\alpha} & \text { if } \alpha \in i\left(\left\langle x_{\alpha}\right\rangle\right) \cap C, \\ p_{\alpha} & \text { otherwise. }\end{cases}
$$


Then $\left\langle z_{\alpha}\right\rangle \in \sigma_{A}^{n}$, and hence $C_{\left\langle z_{\alpha}\right\rangle} \subset C_{n+1}$. Further, since $x_{\alpha}=z_{\alpha}$ for each $\alpha$ in $C_{\left\langle z_{\alpha}\right\rangle}$, it follows that $\left\langle x_{\alpha}\right\rangle \in S_{\left\langle z_{\alpha}\right\rangle}$. But this is a contradiction since $S_{\left\langle z_{\alpha}\right\rangle} \cap Y=\varnothing$. Hence it is proved that $t\left(s\left(\left\langle x_{\alpha}\right\rangle\right)\right) \cap C \neq \varnothing$ for each $\left\langle x_{\alpha}\right\rangle$ in $Y$. Then, since $\mathcal{E}$ is a disjoint family of countably infinite subsets of $A$, the cardinality of $Y$ must be at most $\boldsymbol{\aleph}_{0}$. But this contradicts Lemma 2.

Since $\left|Z_{A}\right| \leqslant|\mathscr{F}(A)| \cdot \sup \left\{\left|\Pi\left\{X_{\beta}^{\prime}: \beta \in B\right\}\right|: B \in \mathscr{F}(A)\right\}=|A| \cdot \boldsymbol{\aleph}_{0}$, if $|A|=\boldsymbol{\aleph}_{1}$, then $\left|Z_{A}\right| \leqslant \aleph_{1}$. Further, the cardinality of every nonrealcompact space is not less than $\aleph_{1}$. Hence we have the following conclusion.

THEOREM. There is a space $X$ with the following properties:

(1) $|X|=\boldsymbol{\aleph}_{1}$,

(2) every dense subspace of $X$ is not realcompact.

COROLlARY. $\mathcal{Q} \Re$ is not closed-hereditary.

\section{REFERENCES}

1. R. L. Blair and A. W. Hager, Extensions of zero-sets and of real-valued functions, Math. Z. 136 (1974), $41-52$

2. R. L. Blair, Spaces in which special sets are z-embedded, Canad. J. Math. 28 (1976), 673-690.

3. E. V. Ščepin, On topological products, groups, and a new class of spaces more general than metric ones, Dokl. Akad. Nauk SSSR 226 (1976), 527-529.

4. T. Terada, Note on z-, $C^{*}$-, and C-embedding, Sci. Rep. Tokyo Kyoiku Daigaku Sect. A 13 (1975), 129-132.

5. __ Dense subspaces of topological spaces (submitted).

Department of Mathematics, Faculty of Engineering, Yokohama National University, 156, HODOGAYA, YOKOHAMA, JAPAN 